\title{
EFEITOS DA SUBSTITUIÇÃO DO MILHO PELA RASPA DE MANDIOCA NA ALIMENTAÇÃO DE FRANGOS DE CORTE, DURANTE AS FASES DE ENGORDA E FINAL ${ }^{1}$
}

\author{
Effect of substituition of corn to cassava meal in diets of growing and finishing broiler
}

\author{
Germano Augusto Jerônimo do Nascimento ${ }^{2}$, Fernando Guilherme Perazzo Costa ${ }^{3}$, \\ Valene da Silva Amarante Júnior ${ }^{4}$, Leilane Rocha Barros ${ }^{2}$
}

\section{RESUMO}

Objetivou-se com este trabalho avaliar a substituição do milho por raspa de mandioca nas rações de frangos de corte, durante as fases de engorda e final. O delineamento utilizado foi o inteiramente casualizado, com seis tratamentos representados pela raspa de mandioca (0, 5, 10, 15, 20 e 25\%) em substituição ao milho, seis repetições e 25 aves por repetição. No final de cada fase, foram analisados o consumo de ração (CR), ganho de peso (GP) e conversão alimentar (CA). Após serem feitas as análises estatísticas e interpretado os resultados, observou-se na fase de engorda e final, efeito quadrático para ganho de peso e conversão alimentar. Quanto aos níveis de raspa de mandioca adequados de acordo com cada fase de criação, pode-se concluir que para a fase de engorda, recomenda-se 10,24\% de raspa de mandioca como sucedâneo ao milho, sem que ocorram prejuízos no desempenho dos animais e para a fase final não se recomenda a adição desse subproduto às rações de frangos de corte, uma vez que ocorre diminuição no ganho de peso e piora na conversão alimentar.

Termos para indexação: Alimentação alternativa, aves, desempenho.

\begin{abstract}
This study was carried out with the objective to evaluate the substitution of the corn for cassava meal in broilers diets in the growing and finishing phases. A completely randomized design, with six treatments representing cassava meal $(0,5,10,15$, 20 and 25\%) in replacing corn, six replicates and 25 broilers per replicate, was used. At the end of each phase, were analyzed feed intake (FI), weight gain (WG) and feed conversion (FC). The analysis of the data shown quadratic effect for growing and final phases. According to the statistical analysis the adequate cassava meal levels for each phase was determined. It was conclude that, in the growing phase, the broiler shown that up to $10.24 \%$ of corn should be replace by cassava meal , without affect the broilers performance, therefore for the final phase, any substitution level of cassava meal should be recommended due to reduction on the performance with the use of this manioc by-product.
\end{abstract}

Index terms: Alternative feeding, broiler, performance.

(Recebido para publicação em 12 de março 2004 e aprovado em 3 de agosto de 2004)

\section{INTRODUÇÃO}

$\mathrm{Na}$ avicultura atual, a alimentação representa aproximadamente $70 \%$ do custo de produção, sendo, portanto, fator de grande importância e preocupação para os produtores e nutricionistas.

A especialização requerida pelas atuais linhagens de frangos de corte deve ser acompanhada por um equilíbrio ideal da dieta, quanto à qualidade da matériaprima, do aspecto físico da ração e quanto ao atendimento dos requerimentos nutricionais mínimos para o máximo desempenho (TEIXEIRA, 1998).

Desta maneira, novas pesquisas têm sido desenvolvidas com o objetivo de determinar as melhores opções de utilização de alimentos alternativos energéticos e protéicos, os quais devem propiciar um bom desempenho produtivo e reprodutivo das aves, reduzindo o custo de alimentação e resultando conseqüentemente em maior lucratividade ao produtor.

A mandioca é um dos alimentos alternativos utilizados na alimentação animal, e pode gerar vários tipos de subprodutos. Geralmente utiliza-se para as aves, a raspa da raiz integral, que apresenta um nível energético em torno de $3138 \mathrm{Kcal}$ de EM/Kg (ROSTAGNO et al., 2000), podendo desta forma ser utilizada como sucedâneo do milho em rações para aves.

Portanto, este trabalho teve como objetivo avaliar o efeito da substituição do milho pela farinha de raspa de mandioca em até $25 \%$ nas rações de frangos de corte, durante as fases de engorda e final.

1. Parte da Dissertação do primeiro autor - PPGZ/CCA/UFPB - Areia, PB.

2. Aluno do Programa de Pós-Graduação em Zootecnia do CCA/UFPB - Areia, PB

3. Professor do DZ/CCA/UFPB - Areia, PB - fperazzo@cca.ufpb.br

4. Aluno do Programa de Doutorado Integrado em Zootecnia (UFC/UFRPE/UFPB) - Areia, PB. 


\section{MATERIAL E MÉTODOS}

O experimento foi conduzido no Módulo de Avicultura do Departamento de Zootecnia, do Centro de Ciências Agrárias da Universidade Federal da Paraíba, no município de Areia - PB, nos meses de dezembro de 2002 e janeiro de 2003. As aves foram criadas até 21 dias de idade em locais favoráveis de acordo com o manejo e nutrição. Os boxes apresentavam dimensões de 1,40 x $1,80 \mathrm{~m}$, com piso de cimento e cobertos com bagaço de cana-de-açúcar, sendo utilizado comedouro tubular e bebedouro pendular para cada boxe.

As fases estudadas foram engorda (22 - 35 dias) e final (36 - 42 dias). Foram utilizados 900 frangos de corte, machos, da linhagem Ross, que receberam seis diferentes tipos de rações, formuladas para atender às exigências nutricionais, segundo Rostagno et al. (2000). Os animais apresentaram pesos médios iniciais de 0,727 e $1,593 \mathrm{~kg}$, para as fases engorda e final, respectivamente.
O delineamento experimental utilizado foi o inteiramente casualizado (DIC), com seis tratamentos representados pela raspa de mandioca $(0,5,10,15,20$ e $25 \%$ ) em substituição ao milho nas rações experimentais (Tabela 1 e 2), com seis repetições e 25 aves por unidade experimental, totalizando 900 aves. As rações foram elaboradas de acordo com a composição química e nutricional da raspa de mandioca integral indicada na Tabela Brasileira (ROSTAGNO et al., 2000). Em seguida, foram enviadas amostras para o Laboratório de Nutrição e Análise de Alimentos do DZ/CCA/UFPB, onde confirmou-se os valores calculados.

Os parâmetros analisados foram consumo de ração, ganho de peso e conversão alimentar. As análises estatísticas das características avaliadas foram realizadas pelo Sistema de Análises Estatísticas e Genéticas (SAEG-8.0), desenvolvido pela UFV (1999), e as estimativas do nível de raspa de mandioca em substituição ao milho, por meio do modelo de regressão.

TABELA 1 - Composição percentual das rações experimentais utilizadas durante o período de 22 a 35 dias de idade dos frangos de corte.

\begin{tabular}{|c|c|c|c|c|c|c|}
\hline \multirow{2}{*}{ INGREDIENTES } & \multicolumn{6}{|c|}{ TRATAMENTOS } \\
\hline & $\mathbf{T 1}$ & $\mathbf{T 2}$ & T3 & T4 & T5 & T6 \\
\hline Milho grão & 64,823 & 58,364 & 51,909 & 45,450 & 38,992 & 32,533 \\
\hline Soja farelo $46 \%$ & 20,349 & 21,411 & 22,478 & 23,544 & 24,607 & 25,674 \\
\hline Soja integral extrusada & 6,000 & 6,000 & 6,000 & 6,000 & 6,000 & 6,000 \\
\hline $\begin{array}{l}\text { Farinha carne e ossos } \\
40 \%\end{array}$ & 4,998 & 5,044 & 5,090 & 5,136 & 5,180 & 5,227 \\
\hline Óleo de soja & 2,262 & 2,666 & 3,074 & 3,482 & 3,890 & 4,298 \\
\hline Calcário & 0,410 & 0,376 & 0,333 & 0,297 & 0,258 & 0,221 \\
\hline Sal comum & 0,280 & 0,274 & 0,272 & 0,266 & 0,262 & 0,254 \\
\hline DL-Metionina (99\%) & 0,200 & 0,209 & 0,215 & 0,222 & 0,227 & 0,235 \\
\hline L-Lisina HCL $(78,4 \%)$ & 0,187 & 0,161 & 0,137 & 0,113 & 0,090 & 0,067 \\
\hline Colina 70\% & 0,035 & 0,035 & 0,035 & 0,035 & 0,035 & 0,035 \\
\hline $\begin{array}{l}\text { Raspa de mandioca inte- } \\
\text { gral }\end{array}$ & 0,000 & 5,000 & 10,000 & 15,000 & 20,000 & 25,000 \\
\hline S.Vitamínico+Mineral ${ }^{3}$ & 0,400 & 0,400 & 0,400 & 0,400 & 0,400 & 0,400 \\
\hline Allzyme-Vegpro ${ }^{4}$ & 0,050 & 0,050 & 0,050 & 0,050 & 0,050 & 0,050 \\
\hline Banox $^{2}$ & 0,010 & 0,010 & 0,010 & 0,010 & 0,010 & 0,010 \\
\hline TOTAL & 100,00 & 100,00 & 100,00 & 100,00 & 100,00 & 100,00 \\
\hline
\end{tabular}


TABELA 1 - Continuação...

\begin{tabular}{lcccccc}
\hline \multicolumn{1}{c}{ INGREDIENTES } & \multicolumn{7}{c}{ TRATAMENTOS } \\
\hline & T1 & T2 & T3 & T4 & T5 & T6 \\
\hline Nutrientes Calculados1 & & & & & & \\
\hline Cálcio (\%) & 0,970 & 0,970 & 0,970 & 0,970 & 0,970 & 0,970 \\
Energia Met. (Kcal/Kg) & 3150 & 3150 & 3150 & 3150 & 3150 & 3150 \\
Fibra bruta (\%) & 2,909 & 2,846 & 2,784 & 2,722 & 2,659 & 2,597 \\
Fósforo Disponível (\%) & 0,410 & 0,410 & 0,410 & 0,410 & 0,410 & 0,410 \\
Lisina - Total (\%) & 1,100 & 1,100 & 1,100 & 1,100 & 1,100 & 1,100 \\
Met + Cis - Total (\%) & 0,820 & 0,820 & 0,820 & 0,820 & 0,820 & 0,820 \\
Metionina - Total (\%) & 0,499 & 0,503 & 0,508 & 0,512 & 0,517 & 0,521 \\
Proteína bruta (\%) & 19,500 & 19,500 & 19,500 & 19,500 & 19,500 & 19,500 \\
Sódio (\%) & 0,180 & 0,180 & 0,180 & 0,180 & 0,180 & 0,180 \\
Treonina - Total (\%) & 0,724 & 0,727 & 0,729 & 0,732 & 0,734 & 0,736 \\
Triptofano - Total (\%) & 0,211 & 0,216 & 0,220 & 0,224 & 0,229 & 0,233 \\
\hline
\end{tabular}

${ }^{1}$ Valores calculados de acordo com ROSTAGNO et al. (2000).

${ }^{2}$ Antioxidante

${ }^{3}$ Níveis de suplementação de Vitaminas Minerais e Aditivos (quantidade por Kg/ração): Vit.A, 10.000 UI; Vit. $D_{3} 2.000$ UI; Vit.E, 30 UI; Vit.B , $_{2}$ mg; Vit.B 6 , 3 mg; Ac. Pantotênico, 12 mg; Biotina, 0,1 g; Vit.K 3 , 3 mg; Ácido fólico, $1 \mathrm{mg}$; Ácido nicotínico, $50 \mathrm{mg}$; Coxistac 12 (Salinomicina 12\%), $66 \mathrm{mg}$; $15 \mathrm{mg}$, cloreto de colina (60\%), 6 mg; BHT, 1 mg; Vit.B ${ }_{12}$, 0,015; Selênio, 0,25 mg; Manganês, 106mg; Ferro, 100 mg; Cobre, 20 mg; Cobalto, 2 mg; Iodo, $2 \mathrm{mg}$ e Excipiente q.s.p. 1.000 g.

${ }^{4}$ Enzima fornecida (Alttech/Ltda)

TABELA 2 - Composição percentual das rações experimentais utilizadas durante o período de 36 a 42 dias de idade dos frangos de corte.

\begin{tabular}{lcccccc}
\hline \multirow{2}{*}{ INGREDIENTES } & \multicolumn{7}{c}{ TRATAMENTOS } \\
\cline { 2 - 7 } & T1 & T2 & T3 & T4 & T5 & T6 \\
\hline Milho grão & 59,289 & 52,830 & 46,372 & 39,911 & 33,455 & 26,996 \\
Soja farelo 46\% & 14,805 & 15,870 & 16,933 & 18,000 & 19,065 & 20,127 \\
Milho sub. prod. industrial & 9,000 & 9,000 & 9,000 & 9,000 & 9,000 & 9,000 \\
Soja integral extrusada & 7,500 & 7,500 & 7,500 & 7,500 & 7,500 & 7,500 \\
Farinha carne e osson 40\% & 4,472 & 4,516 & 4,561 & 4,605 & 4,650 & 4,699 \\
Óleo de soja & 3,377 & 3,787 & 4,194 & 4,600 & 5,009 & 5,416 \\
Calcário & 0,405 & 0,366 & 0,327 & 0,292 & 0,254 & 0,216 \\
\hline
\end{tabular}

Ciênc. agrotec., Lavras, v. 29, n. 1, p. 200-207, jan./fev. 2005 
TABELA 2 - Continuação ...

\begin{tabular}{|c|c|c|c|c|c|c|}
\hline \multirow[t]{2}{*}{ INGREDIENTES } & \multicolumn{6}{|c|}{ TRATAMENTOS } \\
\hline & T1 & $\mathbf{T} 2$ & T3 & T4 & T5 & T6 \\
\hline Sal comum & 0,294 & 0,292 & 0,288 & 0,283 & 0,277 & 0,275 \\
\hline DL-Metionina (99\%) & 0,177 & 0,183 & 0,188 & 0,200 & 0,205 & 0,211 \\
\hline L-Lisina HCL $(78,4 \%)$ & 0,194 & 0,172 & 0,150 & 0,122 & 0,100 & 0,076 \\
\hline Colina 70\% & 0,027 & 0,027 & 0,027 & 0,027 & 0,027 & 0,027 \\
\hline Raspa de mandioca integral & 0,000 & 5,000 & 10,000 & 15,000 & 20,000 & 25,000 \\
\hline S.Vitamínico+Mineral ${ }^{3}$ & 0,400 & 0,400 & 0,400 & 0,400 & 0,400 & 0,400 \\
\hline Allzyme-Vegpro ${ }^{4}$ & 0,050 & 0,050 & 0,050 & 0,050 & 0,050 & 0,050 \\
\hline Banox $^{2}$ & 0,010 & 0,010 & 0,010 & 0,010 & 0,010 & 0,010 \\
\hline TOTAL & 100,00 & 100,00 & 100,00 & 100,00 & 100,00 & 100,00 \\
\hline \multicolumn{7}{|l|}{ Nutrientes Calculados ${ }^{1}$} \\
\hline Cálcio (\%) & 0,880 & 0,880 & 0,880 & 0,880 & 0,880 & 0,880 \\
\hline Energia Met. (Kcal/Kg) & 3250 & 3250 & 3250 & 3250 & 3250 & 3250 \\
\hline Fibra bruta (\%) & 2,797 & 2,735 & 2,673 & 2,610 & 2,548 & 2,486 \\
\hline Fósforo Disponível (\%) & 0,380 & 0,380 & 0,380 & 0,380 & 0,380 & 0,380 \\
\hline Lisina - Total (\%) & 1,000 & 1,000 & 1,000 & 1,000 & 1,000 & 1,000 \\
\hline Met + Cis - Total (\%) & 0,750 & 0,750 & 0,750 & 0,750 & 0,750 & 0,750 \\
\hline Metionina - Total (\%) & 0,453 & 0,457 & 0,462 & 0,466 & 0,471 & 0,475 \\
\hline Proteína bruta (\%) & 17,700 & 17,700 & 17,700 & 17,700 & 17,700 & 17,700 \\
\hline Sódio (\%) & 0,180 & 0,180 & 0,180 & 0,180 & 0,180 & 0,180 \\
\hline Treonina - Total (\%) & 0,659 & 0,662 & 0,664 & 0,666 & 0,669 & 0,671 \\
\hline Triptofano - Total (\%) & 0,187 & 0,192 & 0,196 & 0,200 & 0,205 & 0,209 \\
\hline
\end{tabular}

${ }^{1}$ Valores calculados de acordo com ROSTAGNO et al. (2000).

${ }^{2}$ Antioxidante

${ }^{3}$ Níveis de suplementação de Vitaminas e Aditivos (quantidade por Kg/ração): Vit.A, 10.000 UI; Vit.D 2.000 UI; Vit.E, 30 UI; Vit.B 1 , 2 mg; Vit.B 6 , 3 mg; Ac. Pantotênico, 12 mg; Biotina, 0,1 g; Vit.K 3 , 3 mg; Ácido fólico, $1 \mathrm{mg}$; Ácido nicotínico, $50 \mathrm{mg}$; Coxistac 12 (Salinomicina 12\%), $66 \mathrm{mg} ; 15 \mathrm{mg}$, cloreto de colina (60\%), 6 mg; BHT, 1 mg; Vit.B ${ }_{12}$, 0,015; Selênio, 0,25 mg; Manganês, 106mg; Ferro, 100 mg; Cobre, 20 mg; Cobalto, 2 mg; Iodo, 2mg e Excipiente q.s.p. 1.000 g.

${ }^{4}$ Enzima fornecida (Alltech/Ltda) 


\section{RESULTADOS E DISCUSSÃO}

\section{Fase de Engorda (22 a 35 dias de idade)}

A mortalidade durante este período foi de $0,88 \%$. Os níveis de raspa de mandioca em substituição ao milho nas rações influenciaram $(\mathrm{P}<0,01)$ o consumo de ração, ganho de peso e a conversão alimentar dos animais, podendo ser visto nas Figuras 1, 2 e 3 respectivamente.

Na Figura 1 observa-se que o consumo de ração apresentou um efeito quadrático, revelando um menor consumo dos animais ao nível de $12,93 \%$ de raspa de mandioca em substituição ao milho na ração. Acima desse nível houve uma retomada positiva no consumo dos animais, provavelmente devido a uma quantidade maior de óleo de soja nessas rações, o que por sua vez aumenta a palatabilidade das mesmas, concordando com Enriquez e Ross (1967), que ao alimentarem pintinhos com uma ração constituída de $50 \%$ de raspa de mandioca em substituição ao milho, conseguiram obter bons resultados quando a dieta foi suplementada com óleo e metionina.

O NRC (1994) destaca a melhora na palatabilidade e na conversão alimentar e a redução na perda de nutrientes, entre outros, como efeitos benéficos do uso de óleo vegetal e/ou gorduras nas formulações das rações.

Verifica-se na Figura 2 que o nível de melhor desempenho para o ganho de peso compreende-se ao nível de $8,4 \%$ de raspa de mandioca na ração, e a medida que se aumenta a porcentagem de raspa na ração dos animais, ocorre um decréscimo no ganho de peso dos mesmos.
Sabe-se que raspa de mandioca apresenta fatores anti-nutricionais, como os polissacarídeos não amiláceos (PNA), que provocam menor eficiência da dieta, menor ganho diário e diminuição na digestibilidade de diversos nutrientes (BRUFAU et al., 1994), o que apesar de não ter sido mensurado neste experimento, poderia justificar a diminuição do desempenho com o aumento dos níveis de raspa de mandioca integral.

Estes resultados concordam com Osei e Twumasi (1989), os quais observaram que os efeitos negativos no desempenho dos frangos de corte não se evidenciaram até $10 \%$ de raspa em substituição ao milho. Ainda Soares et al. (1968), por meio de análise de regressão constataram que o nível de $10,36 \%$ de substituição foi o que produziu maior ganho de peso dos animais.

Na Figura 3, os dados para conversão alimentar demonstram melhores resultados quando se utiliza o nível de $10,29 \%$ de raspa na ração em substituição ao milho, revelando dessa forma, resultados indesejáveis à medida que se aumenta a porcentagem de mandioca na ração.

Apesar de se verificar aumento na conversão a partir dos $10 \%$ de substituição, alguns autores como Mcmillan e Dudley (1955), verificaram prejuízos econômicos acentuados em relação ao ganho de peso e conversão alimentar, quando as substituições foram acima de $20 \%$, principalmente no período de crescimento dos frangos.

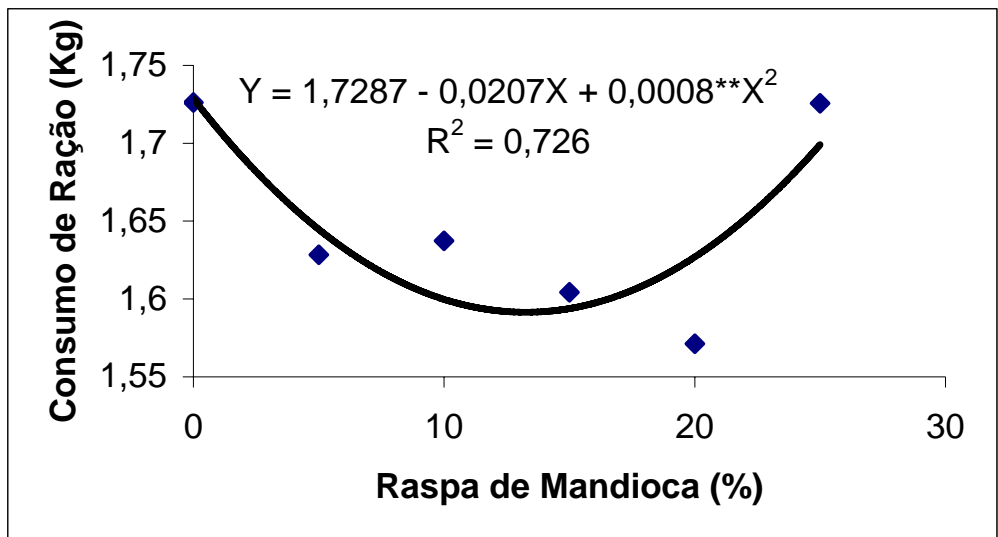

FIGURA 1 - Efeito dos tratamentos sobre o consumo de ração dos frangos de corte, no período de 22 a 35 dias de idade.

Ciênc. agrotec., Lavras, v. 29, n. 1, p. 200-207, jan./fev. 2005 


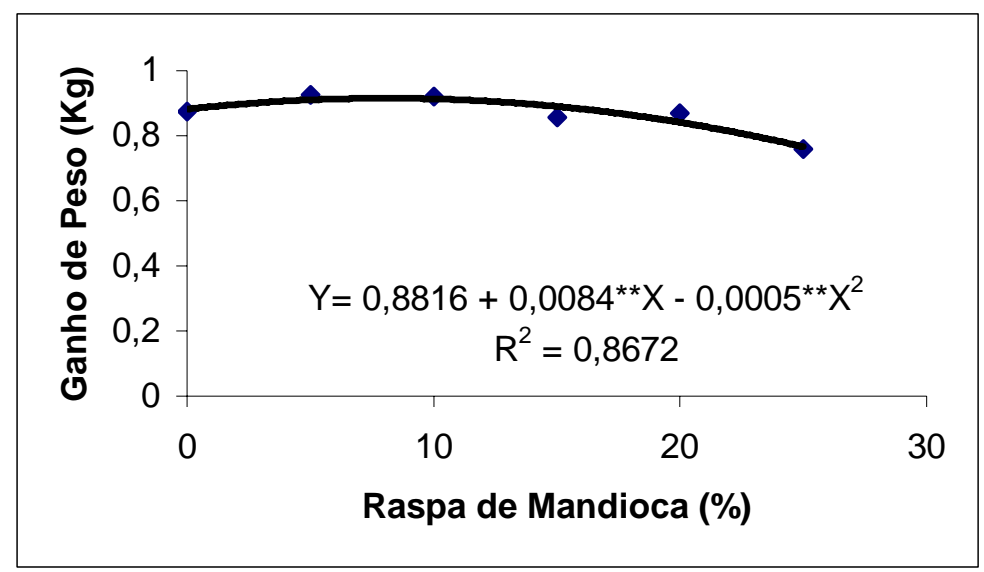

FIGURA 2 - Efeito dos tratamentos sobre o Ganho de Peso dos frangos de corte, no período de 22 a 35 dias de idade.

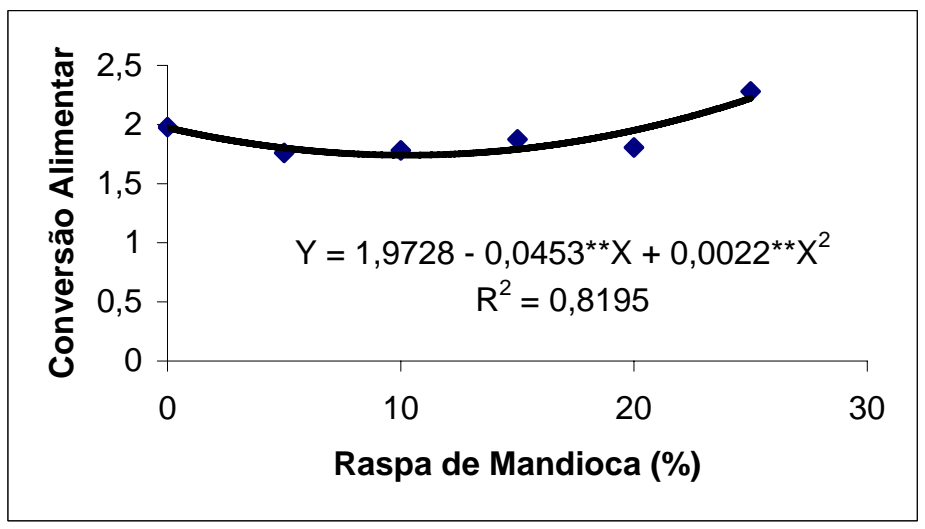

FIGURA 3 - Efeito dos tratamentos sobre Conversão Alimentar dos frangos de corte, no período de 22 a 35 dias de idade.

\section{Fase Final (36 a 42 dias de idade)}

A mortalidade durante este período foi de $0,77 \%$. Os níveis de raspa de mandioca em substituição ao milho nas rações influenciaram $(\mathrm{P}<0,01)$ no ganho de peso e a conversão alimentar dos animais, como pode ser visto nas Figuras 4 e 5 respectivamente, entretanto, não houve efeito significativo sobre o consumo de ração $(\mathrm{P}>0,05)$.

De acordo com Samarasinghe e Wenk (1992), na farinha de raiz de mandioca existem grânulos de amido e polissacarídeos não amiláceos (PNA) que não são digeridos pelas aves, causando flatulência e transtornos digestivos, provocando má absorção dos glicosídeos derivados da sacarose e amido, os quais têm recebido poucas considerações, já que se assume que são integralmente absorvidos (ANDERSON et al., 1981).

Na Figura 4, observa-se que ocorreu efeito quadrático $(\mathrm{P}<0,01)$ com decréscimo no ganho de peso à medida que se aumentou a quantidade de raspa de mandioca na ração, afirmando-se que o pior desempenho para ganho de peso foi ao nível de $15 \%$ de raspa de mandioca em substituição ao milho.

Carrijo et al. (2002), ao utilizarem farelo de raiz integral de mandioca na alimentação de frangos de corte tipo caipira, com quatro níveis de substituição ao milho (0, 45 , 50 e $55 \%$ ), observaram que as aves que receberam rações com esse farelo, apresentaram numericamente peso médio final e ganho de peso maiores do que as aves que 
foram alimentadas com ração sem o referido subproduto, mesmo não apresentando efeito significativo.

Com relação à conversão alimentar (Figura 5), os resultados mostram efeito quadrático $(\mathrm{P}<0,01)$, ocorrendo um acréscimo dessa conversão à medida que se aumenta a quantidade de raspa de mandioca, revelando que a pior conversão alimentar está ao nível de 13,32\% de raspa de mandioca na ração.

Fonseca et al. (1998) observaram que o consumo de ração e a conversão alimentar das aves foi afetado negativamente nos animais que consumiram dieta à base de farinha de raiz de mandioca. Trabalhando com poedeiras que foram alimentadas com rações em que o milho foi substituído por farinha de raspa de mandioca em níveis crescentes de 20, 30, 40 e 50\%, Chou et al. (1974) verificaram que quando suplementaram a ração com aminoácidos sulfurosos, a substituição ao nível máximo não prejudicou o peso corporal, conversão alimentar e mortalidade. Contudo, a produção e tamanho dos ovos foram melhores com alimentação à base de milho.

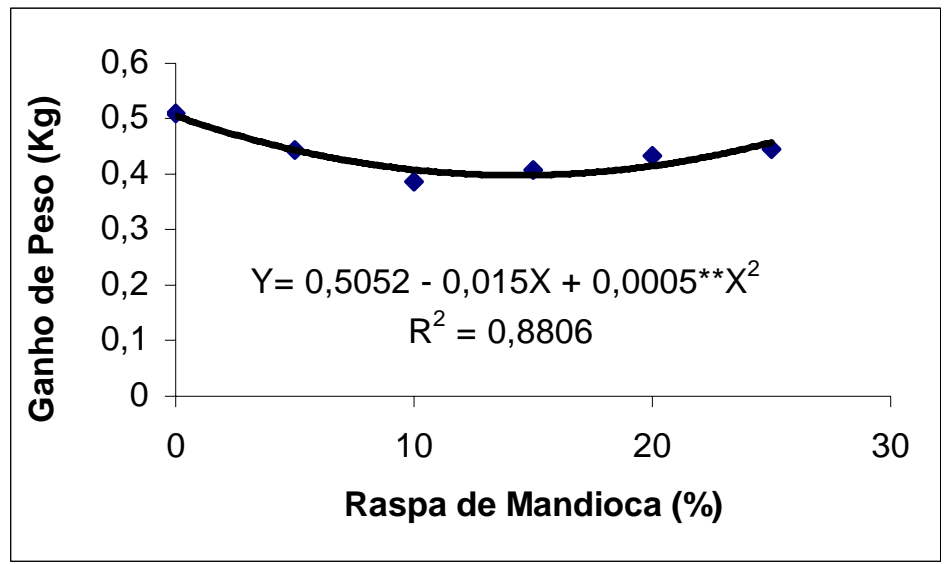

FIGURA 4 - Efeito dos tratamentos sobre o ganho de peso dos frangos de corte, no período de 36 a 42 dias de idade.

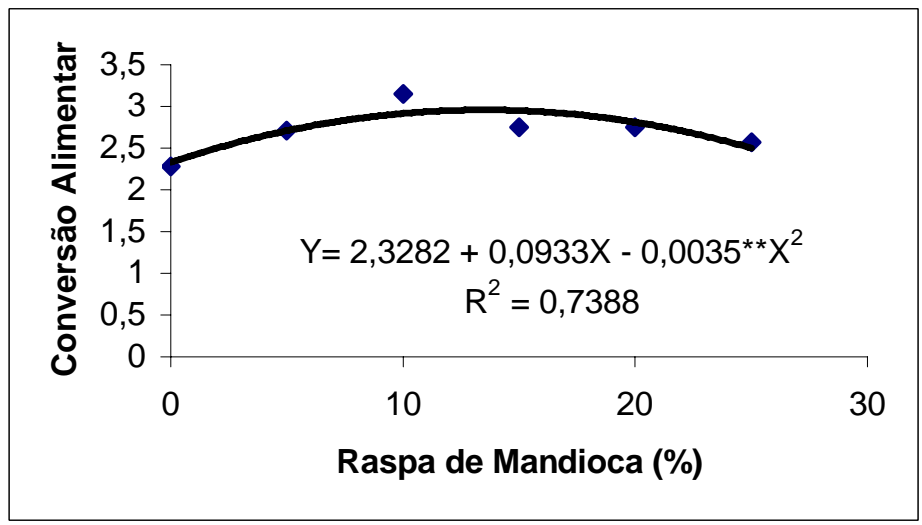

FIGURA 5 - Efeito dos tratamentos sobre conversão alimentar dos frangos de corte, no período de 36 a 42 dias de idade.

Ciênc. agrotec., Lavras, v. 29, n. 1, p. 200-207, jan./fev. 2005 


\section{CONCLUSÕES}

Em consideração à conversão alimentar, recomenda-se utilizar 10,29\% de farinha de raspa de mandioca em substituição ao milho nas rações de engorda dos frangos de corte, enquanto que na fase final, à medida que se aumentam os níveis da farinha, os resultados apresentam-se desfavoráveis.

\section{REFERÊNCIAS BIBLIOGRÁFICAS}

ANDERSON, H.; LEVINE, A. S.; LEVITT, M. D. Incomplete absorption of the carbohydrate in all-purpose wheat flour. Journal Medicinal, New England, v. 304, p. 891-892, 1981.

BRUFAU, J.; PÉREZ-VENDRELL, A. M.; FRANCESCH, M. Papel de la fibra en la alimentacion avícola. In: SIMPÓSIO DE AVICULTURA, 31., 1994, Pamplona. Anais... Pamplona: Seccion Española de la Associacion Mundial de Avicultura Científica, 1994. p. 125-130.

CARRIJO, A. S. et al. Utilização do farelo de raiz integral de mandioca como fonte energética alternativa na engorda de frango tipo caipira. In: REUNIÃO ANUAL DA SOCIEDADE BRASILEIRA DE ZOOTECNIA, 39., 2002, Recife. Anais... Recife: Sociedade Brasileira de Zootecnia, 2002. CD-ROM.

CHOU, K. C.; MULLER, Z.; NAH, K. C. High levels of tapioca-meal in poultry rations. Indian Journal of Animal Science, New Delhi, v. 49, n. 9, p. 697-702, 1974.

ENRIQUEZ, F. Q.; ROSS, E. The value of cassava root meal for chicks. Poultry Science, Ithaca v. 46, p. 622626, 1967.

FONSECA, R. A.; BARBERA, J. B.; OLLÉ, M. F. Farinha de raiz de mandioca, em dietas fareladas e peletizadas de poedeiras semipesadas, com adição de enzimas. In: REUNIÃO ANUAL DA SOCIEDADE BRASILEIRA DE ZOOTECNIA, 35., 1998, Botucatu. Anais... Botucatu: Sociedade Brasileira de Zootecnia, 1998. CD-ROM.

McMILLAN, A. M.; DUDLEY, J. J. Potato meal, tapioca meal and town waste in chicken rations. In:

Feeding poultry. 2. ed. New York: John Wiley \& Sons, 1955. p. 435-43.

NUTRIENT REQUIREMENTS COUNCIL. Nutrient requirements of poultry. 9. ed. Washington: National Academy, 1994. 155 p.

OSEI, S. A.; TWUMASI, J. K. Effects of oven-dried cassava peal meal on the performance and carcass characteristics of Broiler Chickens. Animal Feed Science and Tecnology, Amsterdam, v. 24, n. 3-4, p. 247-252, 1989.

ROSTAGNO, H. S. et al. Tabelas brasileiras para aves e suínos: composição de alimentos e exigências nutricionais. Viçosa: UFV, 2000. 141 p.

SAMARASINGHE, K.; WENK, C. Substitution of cereals by cassava root meal in broiler diets. 1992. $121 \mathrm{f}$. Thesis (Doctor) - INW, ETH, Zurich, 1992.

SOARES, P. R.; CAMPOS, J.; CONRAD, H. J. Farelo integral de raspa de mandioca e farelinho de trigo na alimentação de pintos. Experimentiae, Viçosa, v. 8, n. 4, p. 109-41, 1968.

EIXEIRA, A. S. Alimentos e alimentação dos animais. 4. ed. Lavras: UFLA/FAEPE, 1998. 402 p.

UNIVERSIDADE FEDERAL DE VIÇOSA. Sistema para análises estatísticas e genéticas. Viçosa, 1999. $69 \mathrm{p}$. 